\title{
Persons Versus Brains: Biological Intelligence in Human Organisms
}

\author{
Eric Steinhart
}

Published (2001) Biology and Philosophy 16 (1), 3-27.

\begin{abstract}
I go deep into the biology of the human organism to argue that the psychological features and functions of persons are realized by cellular and molecular parallel distributed processing networks dispersed throughout the whole body. Persons supervene on the computational processes of nervous, endocrine, immune, and genetic networks. Persons do not go with brains.
\end{abstract}

\section{Introduction}

Puccetti (1969) famously argued that brain transplantation preserves the personal identity of the brain-donor: "Where goes a brain, there goes a person." The thesis has been widely discussed. ${ }^{1}$ I argue that persons do not go with their brains. I am concerned only with human persons, ${ }^{2}$ who are naturally realized by (at least parts of) human organisms. ${ }^{3}$

${ }^{1}$ A short list of those who argue that persons go with brains or who advocate brain-based criteria of personal identity includes: Puccetti, 1969, 1970, 1978; Shoemaker, 1970; Green \& Wikler, 1980; Parfit, 1984: sec 89; van Inwagen, 1990: ch. 15. Those who suggest that persons are brains include: Nagel, 1986: 40 - 49. Those who are skeptical that persons go with brains include: Brennan, 1969; Borowski, 1976; Snowdon, 1990, 1991; Harris, 1995; Burke, 1997. The arguments against brain transplants are conceptual; Harris alone raises some physiological worries, but he does not develop them. I am not aware of anyone who outright argues that persons do not go with brains. ${ }^{2}$ Some persons might not have brains or bodies. It has been argued that God, the Devil, angels, demons, extraterrestrials, Neanderthals, dolphins, robots, etc are persons. Those are all non-human persons; I consider only human persons.

3It is sometimes said that the mere logical or conceptual possibility of brain transplants is sufficient to refute bodily criteria of personal identity (see Noonan, 1989: sec. 1.4). The naive view of possibility as "anything goes" must be replaced with clear modal analysis (Lewis, 1973). Accordingly, I suppose that there is some set of logically possible worlds such that (1) each world in that set is physically accessible from this world; (2) each world in that set contains a humanoid species that is the counterpart of the human species in this world; and (3) person-preserving brain transplants are medically possible for the members of those humanoid species. My arguments aim to show that the actual world is not in that set of possible worlds, and the actual human species is not in that set of humanoid species. More precisely: in every possible world that is biologically accessible 
One might immediately object that we cannot know whether persons go with their brains until we have actually performed a human brain or head transplant. The personal identity literature is notorious for its wild thought-experiments. Wilkes (1988) and Baillie (1993: ch. 5) stress the need to focus on real people. Still: head transplant techniques may someday be applied to real people. Robert J. White claims that head transplants will soon be medically feasible (White, 1999; LaFee, 2000). White is the expert on such transplants; his claims are not idle. ${ }^{4}$ We are at least ethically obligated to use the data at our disposal to think seriously about the consequences of such operations. We are obligated to speculate. But I am not interested in groundless thought-experiments. I aim to carefully extrapolate from the available scientific data to the consequences of brain transplantation.

Today the available scientific data argues against Puccetti's thesis: persons do not go with their brains. Many psychological features and functions essential to personhood that were once thought to be realized wholly by physiological structures in the brain are now known to be realized partly by physiological structures outside the brain. One might infer that this makes any discussion of brain transplantation superfluous. However, brain transplantation thought experiments have cast a long anti-biological shadow over the personal identity literature. The thesis that persons go with brains is the major obstacle to biological criteria of personal identity. ${ }^{5}$ If one aims (as I do) to state biological criteria of personal identity, the brain transplant thought experiments must be attacked directly. I

from our world (thta has the same biological laws as our world), persons do not go with brains.

${ }^{4}$ White led the team that successfully transplanted rhesus monkey heads (White et al., 1971). His work has steadily improved since then (White et al., 1996).

${ }^{5}$ I suppose that any Biological Criterion of personal identity is some Bodily Criterion. The Bodily Criterion says (roughly) that persistence of the same human organism (the body) is both necessary and sufficient for persistence of the same human person. The standard argument against the Bodily Criterion is that it is possible for persons to switch bodies, so that persistence of the same organism is not necessary for persistence of the same person and that persistence of something that is not an organism (a soul or a brain) is sufficient for the persistence of the same person. Locke (1959: ch. 27, sec. 15) imagines that the soul of a Prince comes to animate the body of a Cobbler; Locke's story depends on the Cartesian soul/body distinction. Quinton (1962) revives the Lockean version of body-switching and hints that this might be possible via brain transplantation. Shoemaker (1963: ch. 1, sec. 8) naturalizes Locke's story: the brain of Brown goes into the body of Robinson to make Brownson; Brownson is the same person as Brown but the same organism as Robinson. Puccetti $(1969,1970,1978)$ argues for the Brain Criterion: the persistence of the same brain is both necessary and sufficient for the persistence of the same person. Noonan (1989: 1.3-1.4) summarizes the argument from brain transplantation against bodily (hence biological) criteria of personal identity. Johnston (1987) gives an excellent critical evaluation of body-switching. Baillie (1993: 9) gives the sophisticated version of the Brain Criterion. I say human persons do not possibly switch bodies. 
use physiological data to argue against the thesis that persons go with brains: if Brown's bodiless brain is put into Robinson's brainless body to produce Brownson (Shoemaker, 1963: 1.8), the result is neither Brown nor Robinson, but either a hybrid person or an indefinite person.

I suggest that to define the person it is necessary to apply some version of functionalism to the whole human organism. For example: persons are realized by networks of subpersonal agencies themselves realized by cellular and molecular systems throughout the body. So: the identity through time of any person realized by a human organism is the functional persistence of these cellular and molecular systems. Functionalism has gained much of its impetus from computational approaches to cognition. Biologists are right to be skeptical of the utility of computational models, especially insofar as they are based on serial electronic von Neumann machines. But molecular approaches to computing are increasingly gaining biological credibility (Yockey, 1992; Cuthbertson et al., 1996; Tomita et al., 1999; Sipper, 1999). Functionalists often say the mind is the software of the brain; insofar as such metaphors are fruitful, I prefer to say that the person is the software of the whole human organism. If persons are programs, they are biological programs that run on networks of cellular computers that contain networks of molecular computers.

My reasoning proceeds through four stages. First, I examine the argument that persons go with brains. Second, I use recent physiological work to argue that psychological features and functions of persons are realized partly in the brain, and partly in the enteric nervous system, the endocrine system, the immune system, and genetic networks. Third, I look at the psychiatric consequences of brain transplantation. Finally, I conclude that the thesis that persons go with brains fails in favor of a more deeply biological theory of personhood that applies functionalist insights to the whole human organism.

\section{Persons, Psyches, Brains}

\subsection{The Classical Argument that Persons Go with Brains}

Locke defines a person as a "thinking intelligent being, that has reason and reflection, and can consider itself as itself, the same thinking thing, in different times and places" (1959: Bk. II, Ch. 27, 11). Definitions like Locke's are widely accepted; I won't quarrel with them here. Let the term "psyche" designate the whole system of psychological features and functions realized by normal human organisms. The psyche is the Lockean thinking intelligent being. I do not consider the possibility that the psyche is an immaterial Cartesian soul. ${ }^{6}$ I consider the psyche only insofar as it is somehow biologically realized by some part of or by the whole of the human organism.

${ }^{6}$ Descartes says that the soul is an unextended thinking thing. I do not know what that is. Since it does not seem possible to individuate Cartesian souls (Flew, 1976), it is likely that such souls are individuated by (parts of) bodies anyway. There may, however, be 
The classical argument that persons go with brains seems to go like this: (1) the person is the same as the psyche; (2) this psyche is realized wholly by this brain; so, (3) this person is realized wholly by this brain. Equivalently: (1) personal identity is psychological identity; (2) psychological identity is brain identity; so, (3) personal identity is brain identity. Consequently: persons go where their brains go. Identity here is identity through time. The classical argument naturalizes Locke's "thinking intelligent being" by equating it with the brain. I have abstracted this reasoning from the stories ${ }^{7}$ told by philosophers (Shoemaker, 1963; Puccetti, 1969; Parfit, 1984: sec. 89, 1995: 34 - 38). Van Inwagen (1990, ch. 15) has offered slightly different version of the classical argument. Since Mackie (1998) has exposed its fatal physiological flaws, I don't consider it.

I accept the major premise of the classical argument. ${ }^{8}$ I aim to attack the minor premise that the psyche is realized wholly by the brain. One way to attack the minor premise is to allow that the psyche is realized wholly by the brain, but to argue that this psyche is realized by this brain if and only if it is in this body. By "body" I usually mean the part of the organism that is not the brain (what van Inwagen calls the "brain-complement"). Suppose we agree that this person is this psyche, and that this psyche is realized by this brain if it is in this body. Once this brain is separated from this body, what is at issue is the necessity of this body for realizing this person. Anyone who thinks that persons go where brains go has to deny that changing the body of the brain changes the person of the brain. They have to argue that this brain realizes this person even if it is in that body. To argue that persons go with brains, it is has to be argued that the way that brains realize persons is sufficiently invariant through change of body that the same brain realizes the same person in different bodies. However, if this brain realizes this person only if it is in this body, then changing the body of the brain changes the person of the brain. It is possible that the same brain realizes different persons in different bodies. Harris (1995: 59 - 61) attacks the classical argument along these lines. I take that attack much further.

I aim to deny the minor premise by arguing that parts of the psyche are partly realized by cellular and molecular systems not in the brain (Frederickson et al., 1991). ${ }^{9}$ The psyche, consequently, is not wholly realized by the brain. I argue that the person supervenes on four tightly coupled organic systems: the central nervous system (CNS), the enteric

ways to reconcile the biological view of human persons with Aristotelian idea of the soul as either the form of or perhaps the functionality of the living human organism. ${ }^{7}$ Philosophical brain-transplant stories are supposed to appeal to our "intuitions". They are not arguments. They are typically as free from facts as they are from reasoning. ${ }^{8}$ The premise that the person is the same as the psyche is under strong attack: theories of personal identity that do not use psychology seriously weaken the classical identification of the person with the psyche (Olson, 1997). ${ }^{9}$ The volume by Frederickson et al. (1991) focuses "on the unexpected finding that actions on receptors in the periphery may provide long-lasting modulation of complex brain functions such as learning and memory" (p. xiii). 
nervous system (ENS), ${ }^{10}$ the immune system (IS) and the endocrine system (ES). These organic systems are biological information-processing networks. Interactions within and between these systems are computational. The person is realized by (1) molecular interactions among cells within each system (e.g. neurotransmitter interactions between brain neurons); by (2) molecular interactions between cells in distinct systems (e.g. cytokine interactions between brain neurons and IS lymphocytes); and by (3) molecular interactions in regulatory networks inside the cells (e.g. genetic networks). ${ }^{11}$ We know enough about these interactions to show that putting Brown's brain into Robinson's body changes them so much that Brownson is not the same person as Brown.

\subsection{The Brain Transplant as an Exceptional Organ Transplant}

I assume that brain-transplant is whole brain transplant (WBT), and that it transfers only the brain. I do not consider head transplants, but my arguments are telling against them too. I will argue that WBT is not sufficient to transfer any person. In cases of WBT, a donor-person's brain is extracted from his or her body and placed into a recipientperson's body. I make no special assumptions about the recipient body. ${ }^{12}$ Some of my

${ }^{10}$ The enteric nervous system (ENS) is part of the autonomic nervous system (ANS). I could argue more generally that the persistence of the functionality of the ANS is necessary for persistence of the person; but I restrict my focus to the ENS.

${ }^{11}$ The CNS, ENS, IS, and ES form one tightly coupled cellular regulatory network (CRN) in the human organism. The CRN is the parallel distributed processing system in the human organism that most intensely realizes the human psyche. Opening every cell in the organism reveals internal regulatory networks at the molecular level. Every cell contains a genomic regulatory network (GRN). The CNS-ENS-IS-ES cellular regulatory network is a system of computationally coupled GRNs. The CRN pervades the organism: it is surrounded by the cells of other organic systems; it is bathed in a common fluid medium that carries molecular signals. On the level of molecular computation, the organism is a system of computationally coupled GRNs. I refer to the cells not in the CNS-ENS-IS-ES cellular regulatory network as the flesh. The GRNs of cells in the CRN are coupled to one another; the GRNs of cells in the CRN are coupled to the GRNs of cells in the flesh; the GRNs of cells in the flesh are coupled to one another. The total system of coupled GRNs is the molecular regulatory network of the human organism (MRN). The human psyche is distributed over the computations in the MRN. It is surely most intensely concentrated in that region of the MRN whose computations are most intensely cognitive: the genomic regulatory networks of the CNS-ENS-IS-ES cellular regulatory network. The GRNs of the CRN constitute the computational core of the human organism. But the psyche is not restricted to that core. Molecular signalling circuits pass out of cells in the CRN, into the flesh, and return to cells in the CRN. The psyche supervenes on the GRN circuits that pass out of the core into the flesh, and it supervenes (more weakly) on the GRN circuits within the flesh. The psyche pervades every living part of the organism.

${ }^{12}$ The greater the dissimilarity between the donor-body and recipient-body, the harder it will be to preserve the donor-psyche. It's usually assumed that donor and recipient are 
objections are mitigated if the recipient and donor are identical (monozygotic) twins with similar medical histories (Parfit, 1984: sec. 89); but to add that qualification is to affirm the essentiality of far more than the brain. Strictly speaking, WBT does not permit tuning the resultant brain-body composite in order to preserve the donor-psyche. WBT fails exactly to the extent that it requires tuning the recipient-body toward the somatic features of the donor-body (e.g. by adjusting endocrine functions). If any tuning is needed to save the donor-psyche, then WBT fails in favor of a weaker thesis. Of course, that does not prohibit tuning in practice. I assume for the sake of argument what is likely impossible: medical problems involved in WBT are solved engineering problems. I suppose that some donor-person's brain has been transplanted into a recipient-person's body. I suppose the resulting human organism lives. I do not thereby imply that any person survives.

Transplants of hands, hearts, lungs, livers, kidneys, pancreases, intestines, corneas, and blood all preserve the identity of the recipient-organism and the recipient-person. Neural grafts to treat Parkinson's disease (Lindvall, 1990) and neural grafts to repair stroke damage (Bonn, 1998) preserve the identity of the recipient-organism and the recipientperson (Mahowald, 1996); so, transplants of some brain-parts preserve the identity of the recipient-organism and recipient-person. Since the brain contains many small networks whose functions vary only slightly across distinct persons, it is likely that there is some threshold of complexity below which transplants of brain-parts saves the psychological continuity of the recipient-person. Maybe this threshold goes all the way up to the cerebral hemisphere. In any case, brain transplants either whole or partial preserve the recipient-organism. Since hearts, kidneys, etc. are all of the same natural kind (organs), there is a strong inductive argument that WBT preserves the identity of the recipientperson. Since transplanting some brain parts preserves the recipient-person, the induction is even stronger. Yet it is asserted that WBT does not preserve the identity of the recipient-person. Obviously, the whole brain must have exceptional features that invalidate the induction.

According to the classical argument, the brain is an exceptional organ because it wholly realizes the psyche (allegedly, it wholly realized the psyche regardless of the body it lives in). But what explains this realization? The most scientific answer today is that the brain is some kind of biological computer uniquely programmed by its history and genetics to realize some particular person. For example, some have argued that brain is a biological Turing-machine; others have argued that it is more powerful than that. But there are many theories of super-Turing computation (Giunti, 1997), so the theory that the brain is a computer is more general than the theory that it is a Turing-machine. I assume the brain realizes this particular psyche because it is a uniquely programmed biological computer.

\subsection{Physiological Realizations of the Psychological}

adults of the same sex; if they are adults of opposite sex, there are many more problems. I don't deal with those extra problems here. 
I take a functionalist approach to the physiological realization of psychology. In particular, homuncular functionalism (Dennett, 1978) seems best suited to the kind of psyche-body relations I believe are consistent with contemporary physiology. For example, Lycan $(1981,1987,1991)$ resolves complex psychological processes into ever simpler functions performed by subpersonal agencies. Although it has been assumed that these subpersonal agencies are realized wholly by the brain, I argue instead that they are realized by cellular and molecular networks that criss-cross many organ boundaries.

I assume that psychological functions and features have properly psychological definitions, that is, definitions that employ no physiological terms, but only semantic, information-theoretic, behavioral-dispositional, or computational terms. I suppose, for instance, that features like memory, and functions like concept-formation and inference, all have properly psychological definitions. If a psychological feature or function is defined in semantic or information-theoretic terms, then we are free to look for it anywhere in the organism.

For example, many theories of personal identity say that learned memories play an essential role in the individuation of persons (Perry, 1975: pt. II). Here, for the sake of illustration, is a simplistic definition of learning in properly psychological terms: learning is the formation of any novel persistent stimulus-response association (where stimulus is any physical input to the organism and response is any behavioral output). The definition is not prejudiced either for or against any organic system. Equipped with such a definition, empirical physiology decides where and how learning occurs in the body. The definition entails that learning occurs during at least: (1) changes in the synapses of brain neurons; (2) changes in the synapses of enteric nervous system neurons; and (3) changes in the VDJ genes of immune system B-cells and T-cells. ${ }^{13}$ If anything like that definition of learning is true, then it is empirically false that WBT preserves all the memories of a person - for there are memories that are in the body but not in the brain.

\section{Physiological Computation}

\subsection{Computation in the Brain}

Suppose we accept the controversial thesis that psyches are computational processes best described in information-theoretic terms. Cognitive science argues that the brain realizes

${ }^{13}$ Upon first exposure to an antigenic stimulus, the immune system (IS) generates a primary immune response. The IS learns how to form antibodies specifically tailored to destroy that stimulus. It stores the description of those antibodies in the VDJ genes of B- or T-cells. The VDJ genes directly represent the structure of the antigen: they are memories. The next time the body is exposed to that antigen, the IS generates a secondary immune response that is much faster, more powerful, and functionally distinct from the primary immune response. The IS has learned a novel stimulus-response association. 
the psyche because it is a natural information-processor. If some computational theory of the psyche is correct, and if persons are psyches, then persons go with their brains only if the brain is the only organ in the body that performs the computations that constitute the psyche. If there are other information-processing systems in the body, then it is possible that those systems also realize parts or aspects of the psyche, so that WBT transfers only some of the psyche, hence not all of the person. If the psyche is a computational process of the human organism, that fact does not imply that it is restricted to the brain.

The brain has often been treated as if it were a single computer; in reality, it is more like a network of computers. The brain is a network of interconnected nerve cells (neurons). It is a neural network (Rumelhart \& McClelland, 1986). Each neuron is a natural computing agent. Neurons all work in parallel; the functions they perform are distributed over their cooperative and competitive interactions. I use the term parallel distributed processing system (PDP system) generically to cover any densely interconnected parallel computing system whose functions are distributed over the interactions of its processors. ${ }^{14}$ So, neural networks are PDP systems. Neurons interact by exchanging richly-structured electrical and molecular signals. Information is carried by physical features of these signals and is processed by signal transformations. The computational features and functions of PDP networks are typically distributed over the global performance of the whole network. Neural PDP nets are collective at the cellular level. For example, in neural network models of memory, memories are encoded by features of the connections among neurons; but each memory is encoded by features of all the connections (Hinton, 1986). When such systems learn a new memory, many connection features change. The collectivism of neural PDP networks extends below the cell into its molecular processes, which are also organized as PDP networks. While the neuron is often treated as a simple electrical device, synaptic changes involved in learning and memory appear to involve complex molecular events regulated by genetic networks internal to the cell (Neugebauer, 1995).

PDP networks extend far beyond the central nervous system. The molecular messengers of the nervous system are found in other PDP networks in the body (Blalock, 1989). For instance: serotonin, dopamine are used in the nervous and immune systems (Mossner \& Lesch, 1998; Ricci \& Amenta, 1994). Organ boundaries (including the blood-brain barrier) are fences with gates unlocked by molecular keys; the selectivity of those gates facilitates rather than impedes the flow of information. For instance: the immune system

${ }^{14}$ I contrast parallel distributed processing (PDP) systems with centralized systems. PDP must contain many processors. The processors must be sufficiently densely interconnected so that their interactions produce some global features or functions that emerge from their local operations. PDP computation is cooperative, collective, and emergent. Emergent computation has been studied in many models. Examples include: many cellular automata (e.g. the Hodgepodge CA and iterated prisonner's dilemma CAs) and ecological models like Ray's Tierra system. If it is true that individual flies, birds, and fish congregate using only local rules, then swarms are PDP systems insofar as the motion of the swarm as one apparent global unit is the result of the collective local actions of its members. 
makes antibodies to dopamine receptors and serotonin transport molecules; these affect cognition (Kessler \& Shinitzky, 1993; Coplan, 1999). At the molecular and electrical levels, a chain made of interacting nerve cells, immune cells, and endocrine cells may be one circuit. Such chains are not always chains of command in which brain cells are the ruling masters and other cells are the obedient slaves. Functions internal to the brain may be regulated by decisions originating in the immuno-endocrine systems. Insofar as molecular and electrical circuits criss-cross the brain, immune, and endocrine systems, those circuits are loops and chains in large PDP networks that supervene partly on the brain and partly on the endocrine and immune systems. Any psychological features and functions realized by those circuits emerge from and supervene on global activity patterns extending far beyond the brain. Changing any part of the web changes the whole.

There is much computational machinery in the body besides the brain. Many non-brain organic systems perform the same kinds of computations as neural networks, and do so by means of the same kinds of cellular and molecular mechanisms; so, insofar as psychology is computational, those systems also perform the same kinds of psychological functions as brain networks. So either WBT is insufficient, or there is some properly psychological reason for excluding non-brain computations from those that constitute the psyche and person. If the physiologists are right, any such reason specifically formulated to exclude non-brain processes will also exclude brain processes. WBT is insufficient.

\subsection{Computation in the Enteric Nervous System}

Suppose it is argued that the brain bears the psyche because it is a complex neural network whose organization embodies a pattern that is unique and invariant for every person. The brain is not the only complex neural network in the body. The enteric nervous system (ENS) is a complex neural network wrapped around the digestive organs. It is a powerful computational system (Goyal \& Hirano, 1996; Blakeslee, 1996; Gershon, 1998). The ENS structurally and functionally resembles the brain in many ways (Gershon, 1999; Giaroni et al., 1999). ${ }^{15}$ The ENS has been called a "second brain". It contains over 100 million neurons organized into several layered structures. It has sensory neurons, interneurons, and motor neurons. The ENS is a PDP system insofar as global coordinated activity patterns emerge from the local interactions of its cells (Thomas et al., 1999). It is hard to see any scientific reason to deny psychological features and functions to the ENS.

${ }^{15}$ Gershon (1999) and Giaroni et al. (1999) list the following structural and functional similarities between the brain and ENS: the ENS contains several layers; all known classes of neurological messenger molecules (neurotransmitters, neuromodulators, etc.) found in the brain are found in the ENS; the ENS contains glial cells that resemble the astrocytes in the brain; enteric ganglia lack collagen fibers; the ENS is vulnerable to lesions otherwise known to occur only in the brain; the ENS contains intrinsic primary afferent neurons that are multipolar and communicate via slow excitatory synaptic potentials. 
The ENS is sufficiently complex and appropriately organized that the functions it performs deserve to be classified as cognitive. The ENS perceives, processes information, and acts. Afferent ENS neurons are densely distributed throughout the intestinal lining to form a rich sensory field (Miftakhov \& Wingate, 1996; Furness et al., 1997). These neurons are sensitive to mechanical forces like the pressure sensors in the tactile modality and to chemicals like the sensors in the modalities of smell and taste. The ENS perceives the contents of the gut via its afferent neurons much as the brain perceives the world via the cells involved in smell, touch, and taste. ${ }^{16}$ The ENS encodes a rich system of dispositions that associate perceived inputs with actions. Many of the mechanisms that are thought to be involved in higher CNS learning and memory formation are found in the ENS (Giaroni et al., 1999). ${ }^{17}$ Although it is not clear what those mechanisms are doing, it would be surprising if they were not performing cognitive tasks in the ENS that are similar to the tasks they perform in the brain. The ENS is easily far more sophisticated than artificial neural networks that are said by their inventors to perform cognitive tasks. Although it is difficult to know how to properly ascribe epistemic properties to neural networks, I do not think it is unreasonable to say that ENS understands digestion or to say that it knows how to digest food. That knowledge is tacit. The ENS knows how to coordinate the chemical processes involving the stomach, the intestines, the pancreas, and the gall bladder.

The ENS interacts with the brain to regulate digestion. Cellular systems in the ENS and the brain are sufficiently well-coupled to form subpersonal agencies that span both systems. The result is a psychological gut-brain axis internal to the person. The ENS and brain communicate via the vagus nerve and via a variety of chemical messengers. The ENS has a high degree of autonomy within the gut-brain axis, forming an equal and often dominant partner with the brain (Gershon, 1999). Strikingly, the ENS is able to function even when the vagus nerve is cut; in the absence of neural signals from the brain, the ENS is able to regulate digestion. Even more strikingly, peptides secreted by the ENS are able to modulate brain functions involved in memory and learning (Morley \& Flood, 1991). The ENS has many interactions with the immune and endocrine systems. The ENS-IS and ENS-ES axes form non-brain psychological axes in the person.

If neural networks bear the psychological (hence personal) identity of the body, then it would be surprising that a neural network as complex and as highly structured as the ENS carries nothing of the person. It is naturally tempting to argue that the regulation of organic functions like digestion is not relevant to personhood. However, if human persons are necessarily realized by human organisms, then the regulation of organic functions is an essential aspect of human personhood. If neural networks not in the brain bear unique psychological features of the donor-person, then WBT does not transfer the

${ }^{16}$ It could be argued that the contents of the gut remain part of the external world until they are internalized, so that the ENS perceives the external world as much as the eyes do.

${ }^{17}$ Giaroni et al. (1999) provide an extensive comparison (Table 4; pp. 1448 -1449) of neuronal plasticity mechanisms found in both the CNS and ENS. 
whole psyche, and so by the classical argument does not suffice to transfer the donorperson, some of whom stays behind. At best, WBT creates a hybrid person whose psyche is realized in part by the donor-brain and in part by the recipient enteric nervous system.

\subsection{Computation in the Endocrine System}

The brain interacts closely with the endocrine system, the system of glands that regulates body processes via hormones. The endocrine system regulates deeply biological features of human organisms: feeding, fleeing, fighting, and sex. While endocrine glands like the pituitary are in the brain, glands like the thyroid, adrenal, and gonadic (testes or ovaries) are not in the brain. Endocrine functions are organized into regulatory PDP networks involving many feedback loops (Brown, 1994, ch. 8). Endocrine glands secrete hormones that play important roles in mature brain-function (Doraiswamy, 1992; Wallace \& MacCrimmon, 1990) and that regulate many cognitive, emotional and behavioral features of adult humans. It is hard to deny the relevance of these features to personal identity.

WBT does not transfer any endocrine glands not in the brain. Insofar as the hormonal functions of endocrine glands individuate persons by individuating their psyches, WBT fails to transfer the donor-person and fails to eliminate the recipient-person. Any collective or emergent features of endocrine feedback networks are not likely to be preserved when the donor brain is linked to the recipient thyroid, adrenals, and gonads. Hormone therapy tunes the recipient-body toward the donor-body, and so negates WBT, since it concedes that the psyche is partly realized by non-brain endocrine glands. At best, WBT creates a hybrid person whose psyche is realized in part by the donor-brain and in part by the recipient non-brain endocrine system and the recipient enteric nervous system.

\subsection{Computation in the Immune System}

The immune system (IS) is a complex information-processing system (Segel \& Perelson, 1988). The immune system is tightly coupled with the nervous and endocrine systems (Cotman, 1987; Ader, 1991, 1995; Maier \& Watkins, 1998). The IS has about $10^{12}$ cells, while the brain has only about $10^{10}$. The IS is remarkably free from CNS control; it is able to cause cognitive and affective changes in the CNS (Maier \& Watkins, 1999).

The IS is undeniably adaptive: when the IS is initially exposed to an antigen (e.g. smallpox), it responds in one way; but when it is exposed to the same antigen again or to a similar antigen (e.g. cowpox), it responds very differently. The IS acquires immunity. It has been argued that the adaptive abilities of the IS are cognitive (Varela et al., 1988; Levy, 1988; Vertosick \& Kelly, 1991; Joshi, 1996). It is possible to train the IS using Pavlovian classical conditioning (Lysle, 1990; Ader \& Cohen, 1991). Much theoretical work in immunology is heavily computational and borrows extensively from artificial intelligence (Farmer et al., 1986; Rowe, 1994: pt. II; Joshi \& Krishnanand, 1996; Joshi, 
1996). The pattern recognition and classification powers of the IS have inspired computer science (Dasgupta \& Attoh-Okine, 1997). The IS is an extremely complex computing system with many of the hallmarks of intelligence. On the available philosophical and psychological theories of perception, cognition, and action, the IS perceives, learns, recognizes, decides, and acts. For example: on my reading of Dretske (1988), the human IS is a Type III Representational System with beliefs and desires. The computational similarities between the IS and brain justify the inference that if the brain is cognitive, then the IS is too.

Even if one accepts that the computations performed by the IS are as complex and subtle as those performed by the brain, one may be tempted to argue that they are not relevant to personhood. Surprisingly, it will be hard to make such arguments. Immunology has been defined as the science of the difference between self and nonself: "Immunology deals with understanding how the body distinguishes between what is 'self' and what is 'nonself'; all the rest is technical detail" (Benjamini, 1996: 1). The immunological self has been called the "somatic" self. There are many ways to conceptualize the selves associated with human persons (Neisser, 1993). If human persons are necessarily realized by human organisms, then the persistence of the somatic self seems necessary for the persistence of any other selves. ${ }^{18}$ Since there is some controversy about some aspects of the somatic self (Tauber, 1994; Silverstein \& Rose, 1997), I will focus on one aspect of the somatic self that is uncontroversial and that seems relevant to personal identity.

One argument for WBT goes like this: a person is identified by memories; memories are realized exclusively by the brain; WBT transfers the brain, so it transfers the memories of the brain, so it transfers the person identified by those memories. The argument depends on the assumption that all memories are realized neurally in the brain. Neural networks are not the only mnemonic systems in the human organism. Acquired immunity is based on immunological memory (IM; Benjamini, 1996: ch. 6, 105-106, 147-148). I suspect many will be skeptical that the IS has anything like memories, since the conscious ego does not seem to have introspective access to those memories. But the conscious ego does not have introspective access to many neural memories; moreover, much psychophilosophical work on amnesia, self-deception, akrasia, blindsight, and confabulation challenges the authority of the conscious ego to constitute the person. In any case, IM shares two essential features with neural memory: it is associative and contentaddressable (Smith, 1998, ch. 6). It is hard to find any cognitive, semantic,

${ }^{18} \mathrm{Of}$ particular interest is the role of the somatic self in the constitution of the "ecological self" and the "interpersonal self" (Neisser, 1993). The immune system appears to play a signficant role in human mate selection. The HLA gene complex encodes the unique IS profile of the somatic self. The HLA complex also encodes molecules secreted in sweat and urine. Human females appear highly sensitive to the HLA-profiles found in the sweat of human males, sexually prefering males whose HLA-profiles differ maximally from their own (Ferstl et al, 1991; Wedekind \& Furi, 1997; Penn \& Potts, 1998). See the articles in Genetica 1998-99 104 (3). It would be fascinating to extend J. J. Gibson's notions of ecological cognition to the IS in its interaction with other people and the world. 
computational, or behavioral-dispositional reasons to exclude IM from personal identity that do not exclude neural memory as well.

IM records the exposure of the body to antigens. Immune responses to antigens involve many kinds of cells; some of these cells are B-lymphocytes (B-cells). After primary exposure to an antigen, those B-cells that respond to it undergo a learning process called affinity maturation. The B-cells that originally responded to the antigen are rapidly evolved via mutation and selection into a new population of B-cells finely-tuned against that specific antigen. In computational terms, the IS runs a genetic algorithm (Goldberg, 1989). Memory of the antigen is encoded in the new DNA (the VDJ genes) of the tuned B-cells. It is easy to define reference for IM memories: the DNA in the VDJ genes in Bcells is a string of symbols that determines the protein structure of an antibody; the antibody is effective against its antigen because they fit like a lock and key. IM has impeccable semantics: it explicitly models correspondence and causal theories of reference.

Affinity maturation forms short-term memories; long-term memory in the IS is not wellunderstood. One hypothesis is that some finely-tuned B-cells become dormant, longlived memory cells. Another hypothesis is that groups of IS cells excite or inhibit one another's actions (much like neurons), thus forming nodes in a PDP immune network (Jerne, 1974; Perelson, 1989; Varela \& Coutinho, 1990; Takumi \& De Boer, 1996; Leon et al., 1998). Immune networks have been compared with neural networks (Dasgupta, 1997). Principles of the immune system have been used to develop theories of neural nets (Hoffman, 1986); principles of neural nets have been used to develop theories of immunity. For instance, neural networks known as self-organizing Kohonen nets have been used to model the IS processes whereby some IS cells develop specific adaptive responses to particular antigens (Roshi, 1996). Immune network theory is controversial; what remains certain is that the IS is an extremely complex computational system, possibly more complex than the brain.

However it is realized, IM is biographical: it records the unique biological history of each human organism. Since IM shares essential semantic and computational features with neural memory, properly psychological arguments against IM will be effective against neural memory too. If it is necessary to preserve memory to transfer the person, then WBT fails, since it does not save IM. At best, WBT creates a hybrid person whose psyche is realized in part by the donor-brain and in part by the recipient immune system, the recipient non-brain endocrine system, and the recipient enteric nervous system.

\subsection{Computation in Genetic Networks}

Powerful molecular computational mechanisms exist in cells. These networks are unique to the organism. Chemical reactions in and between cells interact to form holistic signaling networks with emergent properties (Bhalla \& Iyengar, 1999). Bhalla \& Iyengar argue that molecular networks store data in feedback loops resembling the flip-flops used in digital computers; if they are right then memories unique to the organism exist in its 
molecular signaling networks. The molecular signaling networks within and between cells may constitute a semiotic system with complex syntactic and semantic properties (Mayer \& Baldi, 1991; Ji, 1997). Cells contain PDP networks that compute at the molecular level. The fact that DNA can be programmed to solve hard combinatorial problems (Adleman, 1994; Beaver, 1995) shows that molecular computing is not merely metaphorical.

Molecules like DNA, RNA, and proteins function as symbols whose shapes determine their semantic interactions. It is not hard to map molecular processes in the cell onto the logical operations of the propositional calculus: an active molecule is a true proposition; chemical reactions are logical operations. For instance, suppose that gene $\mathrm{G}$ is activated (switched on) by molecular trigger $\mathrm{T}$ or $\mathrm{S}$; when $\mathrm{G}$ is on, it produces an RNA molecule $\mathrm{R}$, which produces protein $\mathrm{P}$. Logically, this is: ( $\mathrm{G}$ and ( $\mathrm{S}$ or $\mathrm{T})$ ) implies $\mathrm{R} ; \mathrm{R}$ implies $\mathrm{P}$. When chemical interactions are mapped onto the propositional calculus, cells look much like inference engines of classical AI - they look much like rule-based expert-systems. Many arguments were made for the intelligence of expert systems far simpler than cells. ${ }^{19}$ If those arguments were sound, then some (small) intelligence emerges from cells.

Since all the molecules in a cell are active simultaneously, cellular logic is more like a PDP network than a serial rule-based expert system. Genes interact to form holistic dynamical systems with emergent properties: genetic networks or genomic regulatory networks (McAdams \& Shapiro, 1995; Smolgyi \& Sniegoski, 1996; Yuh, 1998). Just as cells (neurons, lymphocytes, and endocrine cells) excite and inhibit one another's actions, so genes excite and inhibit one another's actions. Strikingly, genetic networks resemble logic-circuit diagrams or flowcharts for procedural computer programs. They are boolean switching networks - just like the old McCulloch-Pitts (1943) model of the brain. I doubt that the architecture of genetic networks suffices to truly call their computations cognitive. In terms of homuncular functionalism, the genetic networks internal to cells are the lowest level homunculi; they are the mindless automata from whose interactions mind emerges. If the human psyche is realized physiologically, then it has to have definite foundations, and the computations on those bottom levels must be realized directly by physical systems. Molecular computation is the lowest level of the human biocomputer; it is where the thinking software emerges from the thoughtless hardware.

If it is necessary to transfer the unique computational features of a person at every level, even the very lowest, in order to transfer the psyche and therefore person, then WBT fails, since it does not transfer the genetic regulatory networks not in the brain. At best, WBT creates a hybrid person whose psyche is realized in part by the genetic networks in the donor-brain and in part by the genetic networks in the recipient-body, particularly those in its enteric nervous system (ENS), immune system (IS), and endocrine system (ES).

${ }^{19}$ If Fodor's (1975) language of though hypothesis is true of the human organism, it is probably most true of the molecular interactions within and among cells. 


\section{Psychiatric Aspects of Brain Transplantation}

\subsection{WBT and Psychological Discontinuity}

If one cannot show that there is psychological continuity between the pre-WBT psyche of the donor-organism and the post-WBT psyche of the recipient-organism, then it is hard to see how one could argue that these distinct organisms bear the same person. As in all other cases of organ transplantation, criteria of personal identity based on bodily continuity favor preserving the person of the recipient-organism, since the recipientorganism survives WBT while the donor-organism either dies or loses all psychological features. Suppose Brown's bodiless brain is put into Robinson's brainless body to produce Brownson. To argue that WBT transfers a person from one body to another is just to argue that the psyche of Brown persists invariant through the change of bodily context. Otherwise either Robinson or no person survives. To defend that persons go with brains is to defend the psychological continuity of Brownson with Brown. Since the Brownson chimera shares no non-brain tissue with Brown, all psychological continuity must be supported by Brownson's brain; that brain must maintain Brown's activity patterns despite its interactions with Robinson's body. I argue that is not biologically possible.

If Brown's bodiless brain is put into Robinson's brainless body, then the cellular regulatory network (CRN) in Brown's brain is mechanically connected to the CRNs in Robinson's spinal cord, autonomic nervous system, ENS, IS and ES. Molecular regulatory networks (MRNs) controlled by two distinct genomes are brought into chemical contact: hormones, cytokines, peptides, antibodies, neurotransmitters, and other chemical signals produced by Robinson's non-brain MRNs interact with Brown's brain MRNs. If the Brownson chimera lives, the result is a hybrid CRN formed partially from Brown's CRN and Robinson's CRN. The hybrid CRN supervenes on a hybrid MRN programmed slightly by Brown' genome but mostly by Robinson's genome. As signals traverse these hybrid networks, what emerges is at worst no psyche at all or at best a hybrid psyche that is neither Brown nor Robinson. If Brownson ever regains consciousness after WBT, he is likely to be vegetative or psychotic; if he recovers from that psychosis, he is not likely to have any psychological continuity with either Robinson or Brown.

\subsection{Psychiatric Consequences of Brain Transplantation}

Brain circuitry is plastic. After implantation, many neural networks in Brown's brain will change to increasingly resemble the ones that were in Robinson's brain. The body-map is a neural network that encodes the structure of the body. The body-map is a plastic network (Martin, 1996: 152-3; Berlucchi, \& Aglioti, 1997). Brown's body-map will change to be more like Robinson's body-map. Since the neural networks in Brown's brain are now regulating visceral processes in Robinson's body, they will change to 
resemble the visceral control networks that were in Robinson's brain. ${ }^{20}$ Sensorimotor coordination networks in Brown's brain will change to resemble those in Robinson's brain: as Brown's brain learns to control Robinson's hands with Robinson's eyes, Brown's neural hand-eye coordination maps reorganize. Brown's facial recognition and voice recognition networks change as he learns to recognize Robinson's face and voice as his own (Leveroni et al., 2000; Sugiura et al. 2000). As all these nets change to resemble those that were in Robinson's brain, the networks that encode Brown's autobiographical memories change (Fink et al. 2000), thereby changing his self-memory system (Conway \& Pleydell-Pearce, 2000). However subtle the changes may be, Brown's brain is becoming like (what used to be) Robinson's brain.

It is strange that mental illness is never mentioned in the philosophical literature on braintransplants. The hybrid organism is simply assumed to be sane. Psychiatry argues against that. ${ }^{21}$ The recipients of transplanted non-brain organs often face profound psychiatric difficulties as they struggle to psychosomatically integrate those organs (Mulsin, 1971; Dubovsky, 1979; Riether, 1990). Transplants can induce dissociative shifts. Body-image and self-concept are often significantly changed by transplants (Castelnuovo-Tedesco, 1973; Chaturvedi \& Pant, 1984). Some recipients of alien hearts insist that they are new people, divorce their spouses, change their families and jobs, and adopt the behaviors they think appropriate for the original bearer of the heart (Bunzel, 1992). WBT has been referred to as a body-transplant: the brain is thought of as remaining the same while every organ of its old body is removed and replaced. But if a body that has just one organ transplanted undergoes serious psychological crisis, with radical changes of its self-conception, then it follows that a body that has every organ removed and replaced will undergo a psychological transformation of self-conception that is complete. These changes of self-conception are changes in the donor-brain, whose psyche dissociates.

${ }^{20}$ Damasio (1999: ch. 5) argues that "the part of the mind we call self [is], biologically speaking, grounded on a collection of nonconscious neural patterns standing for the part of the organism we call the body proper" (p. 134; Damasio's "body proper" is the nonbrain part of the organism). He claims that the higher-level autobiographical self is based on what he calls the "proto-self": "The proto-self is a coherent collection of neural patterns which map, moment by moment, the state of the physical structure of the organism in its many dimensions" (p. 154). Brownson's proto-self will evolve to become like Robinson's proto-self, insofar as Brownson's brain interacts with Robinson's body. ${ }^{21}$ A look at the Diagnostic and Statistical Manual for Mental Disorders, 4th Ed. (DSMIV , 1994) indicates the psychiatric disorders whose conditions are satisfied by WBT. I indicate the disorders by their DSM-IV codes in parentheses. Brownson will suffer from somatoform disorders $(300.81 ; 300.7 ; 300.11 ; 307)$. Brownson will suffer most from all the dissociative disorders: dissociative amnesia (300.12), dissociative fugue (300.13), and depersonalization disorder (300.6). Depersonalization is likely to put the Brownson into a delusional state (e.g. permanent out-of-body experience). Dissociative identity disorder (300.14) is likely to utterly destroy the donor-psyche. I think these disorders suffice to destroy any psychological continuity between Brownson and Brown. 
The psychiatric literature on dissociative disorders suggests that physiological continuity of brain function does not suffice for psychological continuity of brain function. The brain (along with the immune and endocrine systems) is able to switch between distinct activity patterns. ${ }^{22}$ These patterns are known as alters. The alters are the distinct personalities of multiple personality disorder (dissociative identity disorder). Dissociative fugue is far more radical than multiple personality disorder (James, 1983: 369 - 371; Ross, 1994). Since amnesic barriers can separate the memory systems of distinct alters, ${ }^{23}$ one cannot argue that brain continuity entails memory continuity. Since distinct alters can have distinct characters and personalities, one cannot argue that brain continuity entails character or personality continuity. ${ }^{24}$ The shift from one alter to another is physiologically continuous but is not psychologically continuous. If my readings of the psychiatric literature are correct, then the physiological continuity of Brown's brain in Robinson's body does not suffice for the preservation of Brown's memories or personality. If my readings of the neurological literature are correct, then the physiological reorganizations of Brown's brain in Robinson's body do suffice to destroy Brown's memories and personality. The psyche realized by Brown's brain in Brown's body is not psychologically continuous with the psyche realized by Brown's brain in Robinson's body. If psychological continuity is needed to preserve persons, then Brownson is not the same person as Brown.

\subsection{The Psychological History of the Brain-Body Composite}

Suppose that Brown's bodiless brain is put into Robinson's brainless body to make Brownson. Brown's brain does not know how to regulate Robinson's body. Brownson is likely to remain in a vegetative state and to suffer violent seizures until his lower brain activity patterns reorganize into patterns compatible with the feedback from his new body. Assuming he ever wakes up, Brownson is likely to have regressed to a psychotic infantile state in which there is no coherent person. He does not know how to eat; he

\footnotetext{
22Physiological differences among distinct personae in dissociative identity disorder include differences in cerebral electrical activity, regional cerebral blood flow, ocular and visual function, dominant handedness, response to medications, autonomic processes, galvanic skin responses, immune system processes, endocrine system processes (Putnam, 1984; Birnbaum, 1996; Miller, 1992; Hughes, 1990; Putnam, 1990). These differences are not differences in the physiological structure (the hardware) of the cellular regulatory networks (CRNs) so much as they are differences in the computational processes (the software) that supervenes on the signaling patterns in those CRNs.

${ }^{23}$ The distinct patterns in dissociative disorders are known as alters. Alters are typically separated by amnesic barriers. Since alters need not share any common autobiographical memory system, criteria of psychological continuity based on memory (e.g. Locke, (1959: bk. II, ch. 27; Grice, 1941; Quinton, 1962) cannot conclude that alters are continuous.

${ }^{24}$ According to Parfit (1984: 206), psychological continuity requires only presevation of at least one-half of the psychological features and functions found to normally persist from day to day in normal people. Dissociative shifts surely often preserve less than that.
} 
cannot control his bowels. He neither moves nor speaks. Anti-psychotic drugs suffice to retrieve that newborn psyche, so that it can learn to animate its body. Brown's brain changes as Brownson learns to walk and to use his hands. Conflicts between Brownson's memories and all his sensory inputs lead to cognitive dissonance and permanent total amnesia. The infantile Brownson psyche will never recover Brown's autobiographical memories.

Brownson is insane. For years he drifts in and out of deep psychosis. As his cellular and molecular regulatory networks cycle chaotically through semi-stable configurations, many weak alters emerge and dissolve. Although initially Brownson is polyfragmented, alters with characters eventually emerge. One character is selected around which to integrate the others. Meanwhile Brownson suffers from the classical dissociative symptoms: seizures, headaches, blackouts. He has auditory and visual hallucinations of Brown's voice and body-image; he does not know who it is. He has depersonalization episodes; he says he is possessed by demons. He is suicidal. He suffers from borderline personality disorders and sometimes self-mutilates. He spends his time on sedation in restraints.

Hard psychiatric work eventually stabilizes Brownson's new persona. Anything related to Brown triggers Brownson's painful dissociative symptoms. Shown a photo of Brown, he flies into a confused rage then blacks out. He recognizes himself in photos of Robinson, but he feels nothing. He confabulates a past: he was tortured; he hurt his head in an accident. Taken to psychiatrists who do not know about the brain transplant, the diagnosis is always swift and sure: Brownson suffers from some dissociative disorder he is in a protracted fugue state. As time goes by, constant therapeutic work and medication help Brownson adapt to his new life. He is less and less concerned with either Brown or Robinson. He becomes his own person. He gets on with his life.

\section{Conclusion}

Corporate theories of persons go back to Plato's analogy of the soul as a city (Republic, 368c-369b). For Aristotle, Plotinus, Augustine, and Aquinas, the soul was a richly structured entity thought of as a system of abstract biological functions. The nutritive, locomotive, reproductive, and other visceral functions of the human organism were not excluded from the soul, but had citizens rights there. Unfortunately, Descartes reduced the soul to just one part: the rational soul. Soul became mind; psyche became nous, anima became mens. The mind was centered in the brain - indeed, in the pineal gland. The Cartesian reduction of the person to the mind stands behind the reductions of the person to the brain. Today we know that Cartesianism is both psychologically and physiologically unrealistic. To achieve a richer theory of persons, modern antiCartesians have returned to the older corporate theories of the soul. Even in the 19th century, Nietzsche argued for a theory of the person as "social structure of the drives and emotions", and declared against Descartes that "our body is a social structure composed of many souls" (Nietzsche, 1966: secs. 12, 19). Dennett's and Lycan's corporate theories of personhood argue that persons emerge from self-organizing collectives of subpersonal 
agencies. Puccetti's slogan, "Where goes a brain, there goes a person" is among the last gasps of Cartesianism dualism; it is a theory that deserves to be laid to rest in favor of richer and more biologically realistic theories of personhood for the human animals that we are. 


\section{References.}

Ader, R. and Cohen, N.: 1991, "The Influence of Conditioning on Immune Responses", in R. Ader et al. (eds.) (1991), 611 - 646.

Ader, R. et al. (eds.): 1991, Psychoneuroimmunology, 2nd Ed., Academic Press, New York.

Ader, R. et al.: 1995, "Psychoneuroimmunology: Interactions between the Nervous System and the Immune System", The Lancet 345 (14 January), 99 - 103.

Adleman, L.: 1994, "Molecular Computation of Solutions of Combinatorial Problems," Science 266 (11 November), 1021-1024.

Baillie, J.: 1995, Problems in Personal Identity, Paragon House, New York.

Beaver, D.: 1995, "Computing with DNA", Journal of Computational Biology 2 (1), 1-7.

Benjamini, E., Sunshine, G., and Leskowitz, S.: 1996, Immunology: A Short Course, John Wiley \& Sons, New York.

Berlucchi, G. and Aglioti, S.: 1997, "The Body in the Brain: Neural Bases of Corporeal Awareness", Trends in Neuroscience 20 (12), 560-4.

Bhalla, U. and Iyengar, R.: 1999, "Emergent Properties of Networks of Biological Signaling Pathways", Science 283 (15 January), 381 - 387.

Birnbaum, M. et al.: 1996, "Visual Function in Multiple Personality Disorder", Journal of the American Optomology Association 67 (6), 327-334.

Blakeslee, S.: 1996, "Complex and Hidden Brain in the Gut Makes Stomachaches and Butterflies", New York Times (23 January), C1.

Blalock, J. E.: 1989, "A Molecular Basis for Bidirectional Communication between the Immune and Neuroendocrine Systems," Physiological Reviews 69 (1), 1 - 32.

Bonn, D.: 1998, "First Cell Transplant Aimed to Reverse Stroke Damage", The Lancet 352 (11 July), 119.

Borowski, E.: 1976, "Identity and Personal Identity", Mind 85 (340), 481 - 502.

Brennan, A.: 1969, "Persons and their Brains", Analysis 30 (1), 27 - 30

Brown, R.: 1994, An Introduction to Endocrinology, Cambridge University Press, New York, ch. 8. 
Bunzel, B. et al.: 1992, "Does Changing the Heart Mean Changing Personality? A Retrospective Inquiry on 47 Heart Transplant Patients", Quality of Life Research 1 (4), 251 - 256.

Burke, M.: 1997, "Persons and Bodies: How to Avoid the New Dualism", American Philosophical Quarterly 34 (4), 457 - 467.

Castelnuovo-Tedesco, P.: 1973, "Organ Transplant, Body Image, Psychosis", Psychoanalytic Quarterly 42 (3), 349-363.

Chaturvedi, S. and Pant, V.: 1984, "Objective Evaluation of Body-Image of Renal Transplant Recipients", Journal of Psychological Researches 28 (1), 4-7.

Conway, M. \& Pleydell-Pearce, C.: 2000, "The Construction of the Autobiographical Memory System", Psychological Review 107 (2), 261 - 288.

Coplan, J. et al.: 1999, "Plasma Anti-serotonin and Serotonin Anti-idiotypic Antibodies are Elevated in Panic Disorder," Neuropsychopharmacology 20 (4), 386 - 391.

Cordo, P. (ed.): 1995, "Controversies in Neuroscience: Neural Transplantation", Behavioral and Brain Sciences 18 (1), 1 - 97.

Cotman, C. et al. (eds.): 1987, The Neuro-Immune-Endocrine Connection, Demos Publications, New York.

Cuthbertson, R., Paton, R., and Holcombe, M.: 1996, Computation in Cellular and Molecular Biological Systems, World Scientific, River Edge, NJ.

Damasio, A.: 1999, The Feeling of What Happens: Body and Emotion in the Making of Consciousness, Harcourt Brace \& Company, New York.

Dasgupta, D. and Attoh-Okine, N.: 1997, "Immunity-Based Systems: A Survey", Proc.of IEEE Intl. Conf. on Systems, Man, and Cybernetics.

Dasgupta, D.: 1997, "Artificial Neural Networks and Artificial Immune Systems: Similarities and Differences", Proc. of IEEE Intl. Conf. on Systems, Man, and Cybernetics.

Dennett, D.: 1978, Brainstorms, MIT Press, Cambridge, MA.

Doraiswamy, P., Krishnan, K., and Nemeroff, C.: 1992, "Hormonal Effects on Brain Function", in D. Barrow \& W. Selman (eds.) Neuroendocrinology, Williams \& Wilkins, Baltimore, 75-91.

Dretske, F.: 1988, Explaining Behavior, MIT Press, Cambridge, MA. 
DSM-IV: 1994, Diagnostic and Statistical Manual of Mental Disorders, 4th Edition, American Psychiatric Association, Washington, DC.

Dubovsky, S., Metzner, J., and Warner, R.: 1979, "Problems with Internalization of a Transplanted Liver", American Journal of Psychiatry 136, 1090-1091.

Farmer, J., Packard, N., and Perelson, A.: 1986, "The Immune System Adaptation and Machine Learning", Physica 22D, 187 - 204.

Ferstl, R., Eggert, F., Pause, B., Schuler, M. Luszyk, D., Westphal, E., Zavazava, N., Muller-Ruchholtz, W.: 1991, "Immune System Signaling to the Other's Brain: MHCSpecific Body Scents in Humans", in Frederickson (ed.) (1991), 497 - 502.

Fink, G., Martowitsch, H., Reinkemeier, M., Bruckbauer, T., Kessler, J., \& Heiss, W.-D., 1996: "Cerebral Representation of One's Own Past: Neural Networks Involved in Autobiographical Memory", Journal of Neuroscience 16 (13), 4275 - 82.

Flew, A.: 1976, The Presumption of Atheism, Harper \& Row, New York, chs. 8 - 10.

Fodor, J.: 1975, The Language of Thought, Harvard University Press, Cambridge, MA.

Frederickson, R., McGaugh, J., Felten, D.: 1991, Peripheral Signaling of the Brain: Role in Neural-Immune Interactions and Learning and Memory, Hogrefe \& Huber, Toronto.

Furness, J. B., Kunze, W. A. A., Bertrand, P. P., Clerc, N., Bornstein, J. C.: 1998, "Intrinsic Primary Afferent Neurons of the Intestine", Progress in Neurobiology 54, 1 $-18$.

Gershon, M. D.: 1999, "The Enteric Nervous System: A Second Brain", Hospital Practice (Office Edition) 34 (7) (15 July), 31 - 42.

Gershon, M.: 1998, The Second Brain, Harper-Collins, New York.

Giaroni, C., De Ponti, F., Cosentino, M., Lecchini, S., \& Figo, G.: 1999, "Plasticity in the Enteric Nervous System", Gastroenterology 117, 1438 - 1458.

Giunti, M.: 1997, Computation, Dynamics, and Cognition, Oxford University Press, New York.

Goldberg, D.: 1989, Genetic Algorithms in Search, Optimization, and Machine Learning, Addison-Wesley, Reading, MA.

Goyal, R. and Hirano, I.: 1996, "The Enteric Nervous System", New England Journal of Medicine 334 (17) (25 April), 1106 - 1115. 
Green, M. \& Wikler, D.: 1980, "Brain Death and Personal Identity", Philosophy and Public Affairs 9 (2), 105-133.

Grice, H. P.: 1941, "Personal Identity", in J. Perry (ed.) (1975), 73 - 95.

Harris, H.: 1995, "An Experimentalist Looks at Identity", in H. Harris (Ed.) (1995) Identity: Essays Based on Herbert Spencer Lectures Given in the University of Oxford, Oxford University Press, New York, 47 - 63.

Hinton, G. et al.: 1986, "Distributed Representations," in D. Rumelhart \& J. McClelland (eds.), 1986, Parallel Distributed Processing, vol. 1, ch. 3.

Hoffmann, G.: 1986, "A Neural Network Model Based on the Analogy with the Immune System", Journal of Theoretical Biology 122, 33-67.

Hughes, J. et al.: 1990, "Brain Mapping in a Case of Multiple Personality", Clinical Electroencephalography 21 (4), 200-209.

James, W.: 1983, The Principles of Psychology, Harvard University Press, Cambridge, MA.

Jerne, N.: 1974, "Towards a Network Theory of the Immune System", Annals of Immunology (Institute Pasteur) 125C, 373-389.

Ji, S.: 1997, "Isomorphism between Cell and Human Languages: Molecular Biological, Bioinformatic and Linguistic Implications," BioSystems 44, 17 - 39.

Johnston, M.: 1987, "Human Beings", Journal of Philosophy 84, 59 - 83.

Joshi, R. and Krishnanand, K.: 1996, "Probabilistic Learning in Immune Network: Weighted Tree Matching Model", Journal of Computational Biology 3 (1), 143-162.

Joshi, R.: 1996, "A Self-Organizing Cognitive Network of Antibody Repertoire Development", Journal of Computational Biology 3 (4), 529 - 545.

Kessler, A. \& Shinitzky, M.: 1993, "Platelets from Schizophrenic Patients Bear Autoimmune Antibodies that Inhibit Dopamine Uptake," Psychobiology 21 (4), 299 306.

LaFee, S.: 2000, "Pushing Organ Transplants to the Extreme -- and Beyond", The San Diego Union-Tribune, 8 March 2000, Wednesday, E-1.

Leon, K. et al.: 1998, "Natural and Induced Tolerance in an Immune Network Model", Journal of Theoretical Biology 193, 519 - 534. 
Leveroni, C., Seidenberg, M. Mayer, A., Mead, L., Binder, J., \& Rao, S.: 2000, "Neural Systems Underlying the Recognition of Familiar and Newly Learned Faces", Journal of Neuroscience 20 (2), 878 - 886.

Levy, E.: 1988, "Networks and Teleology", in M. Matthen \& B. Linsky (eds.), Philosophy and Biology, University of Calgary Press, Calgary, 159-186.

Lewis, D. (1973) Counterfactuals, Harvard University Press, Cambridge, MA.

Lindvall , O. et al.: 1990, "Grafts of Fetal Dopamine Neurons Survive and Improve Motor Function in Parkinson's Disease", Science 247 (2 February), 574 - 577.

Locke, J.: 1959, An Essay Concerning Human Understanding, Dover Publications, New York. Original work published 1690.

Lycan, W.: 1981, "Form, Function, and Feel", Journal of Philosophy 78 (1), 24 - 49.

Lycan, W.: 1987, Consciousness, MIT Press, Cambridge, MA.

Lycan, W.: 1991, "Homuncular Functionalism Meets PDP", in W. Ramsey, S. Stich, D. E. Rumelhart (eds.), Philosophy and Connectionist Theory, Lawrence Erlbaum Associates, Hillsdale, NJ, 259 - 287.

Lysle, D. et al.: 1990, "Characterization of Immune Alterations Induced by a Conditioned Aversive Stimulus", Psychobiology 18 (2), 220 - 226.

Mackie, D.: 1998, "Going Topless", Ratio 11 (2), 125-140.

Mahowald, M. B.: 1996, "The Brain and the I: Neurodevelopment and Personal Identity," Journal of Social Philosophy 27 (3), 49-60.

Maier, S. \& Watkins, L.: 1998, "Cytokines for Psychologists: Implications of Bidirectional Immune-to-Brain Communication for Understanding Behavior, Mood, and Cognition", Psychological Review 105 (1), 83 - 107.

Maier, S. \& Watkins, L.: 1999, "Bidirectional Communication Between the Brain and the Immune System: Implications for Behavior", Animal Behaviour 57, 741 - 751.

Martin, J.: 1996, Neuroanatomy: Text and Atlas, Appleton \& Lange, Stamford, CT, 152 153.

Mayer, E. and Baldi, J.: 1991, "Can Regulatory Peptides be Regarded as Words of a Biological Language?", American Journal of Physiology 261, G171 - G184;

McAdams, H. and Shapiro, L.: 1995, "Circuit Simulation of Genetic Networks", Science 269 (4 August), 650 - 656. 
McCulloch, W. and Pitts, W.: 1943, "A Logical Calculus of the Ideas Immanent in Nervous Activity", Bulletin of Mathematical Biophysics 5, 115 - 133.

Miftakhov, R. N. \& Wingate, D. L.: 1996, "Electrical Activity of the Sensory Afferent Pathways in the Enteric Nervous System", Biological Cybernetics 75, 471 - 483.

Miller, S. et al.: 1992, "The Psychophysiological Investigation of Multiple Personality Disorder", American Journal of Clinical Hypnosis 35 (1), 47 - 61.

Morley, J. \& Flood, J.: 1991, "Gut Peptides as Modulators of Memory", in Frederickson et al. (eds.) (1991), 379 - 390.

Mossner, R. \& Lesch, K.-P.: 1998, "Role of Serotonin in the Immune System and in Neuroimmune Interactions," Brain, Behavior, and Immunity 12, 249 - 271.

Mulsin, H.: 1971, "On Acquiring a Kidney", American Journal of Psychiatry 127, 11851188.

Nagel, T.: 1986, The View from Nowhere, Oxford University Press, New York.

Neisser, U.: 1993, The Perceived Self: Ecological and Interpersonal Sources of SelfKnowledge, Cambridge University Press, New York.

Neugebauer, A. (ed.): 1995, Macromolecular Interplay in Brain Associative Mechanisms, World Scientific, River Edge, NJ.

Nietzsche, F.: 1966, Beyond Good and Evil, trans. W. Kaufmann, Random House, New York.

Noonan, H.: 1989, Personal Identity, Routledge, London.

Olston, E.: 1997, The Human Animal: Personal Identity without Psychology, Oxford University Press, New York.

Parfit, D.: 1984, Reasons and Person, Oxford University Press, New York.

Penn, D. \& Potts, W.: 1998, "How Do Major Histocompatiblity Complex Genes Influence Odor and Mating Preferences?", Advances in Immunology 69, 411 - 436.

Perelson, A.: 1989, "Immune Network Theory", Immunological Reviews 110, 5-36.

Perry, J. (ed.): 1975, Personal Identity, University of California Press, Berkeley, CA.

Puccetti, R.: 1969, "Brain Transplantation and Personal Identity", Analysis 29 (3), 65-77. 
Puccetti, R.: 1970, "Mr. Brennan on Person's Brains", Analysis 31 (1), 30-32.

Puccetti, R.: 1978, "Borowski on the Relative Identity of Persons", Mind 87 (346), 262 263.

Putnam, F.: 1984, "The Psychophysiologic Investigation of Multiple Personality Disorder", Psychiatric Clinic North America 7 (1), 31-39.

Putnam, F.: 1990, "Differential Autonomic Nervous System Activity in Multiple Personality Disorder", Psychiatry Research 31 (3), 251-260.

Quinton, A.: 1962, "The Soul", in J. Perry (ed.) (1975), 53 - 72.

Ricci, A. \& Amenta, F.: 1994, "Dopamine D5 Receptors in Human Peripheral Blood lymphocytes," Journal of Neuroimmunology 53,1 - 7.

Riether, A.: 1990, "Psychiatric Aspects of Transplantation", in S. Smith (ed.) Tissue and Organ Transplantation: Implications for Professional Nursing Practice, MosbyYear, St. Louis, 117-143.

Roshi, R.: 1996, "A Self-Organizing Cognitive Network of Antibody Repertoire Development", Journal of Computational Biology 3 (4), 529-545.

Ross, C.: 1994, The Osiris Complex: Case-Studies in Multiple Personality Disorder, University of Toronto Press, Toronto, ch. 20.

Rowe, G.: 1994, Theoretical Models in Biology: The Origin of Life, the Immune System, and the Brain, Oxford University Press, New York.

Rumelhart, D. and McClelland, J. (eds.): 1986, Parallel Distributed Processing: Explorations in the Microstructure of Cognition, MIT Press, Cambridge, MA.

Segel, L. and Perelson, A.: 1988, "Computations in Shape Space: A New Approach to Immune Network Theory", in A. Perelson (ed.) Theoretical Immunology (pt. 2, vol. 3), Addison-Wesley, Reading, CA, 321-343.

Shoemaker, S.: 1963, Self-Knowledge and Self-Identity, Cornell University Press, Ithaca, NY.

Shoemaker, S.: 1970, "Persons and their Pasts", American Philosophical Quarterly 7 (4), $269-285$.

Silverstein, A. and Rose, N.: 1997, "On the Mystique of the Immunological Self" and the critical replies to this article in Immunological Reviews 159.

Sipper, M.: 1999, "The Emergence of Cellular Computing", Computer 32 (7), 18 - 26. 
Smith, D., Forrest, S., and Perelson, A.: 1998, "Immunological Memory is Associative", in D. Dasgupta, Artificial Immune Systems and their Applications, Springer-Verlag, New York, ch. 6.

Smolgyi, R. and Sniegoski, C.: 1996, "Modeling the Complexity of Genetic Networks: Understanding Multigenic and Pleiotropic Regulation", Complexity 1 (6), 45 - 63.

Snowdon, P. F.: 1990, "Persons, Animals, and Ourselves", in C. Gill (Ed.) (1990) The Person and the Human Mind, Oxford University Press, New York, 83 - 108.

Snowdon, P.: 1991, "Personal Identity and Brain Transplants", in D. Cockburn (ed.), Human Beings, Cambridge University Press, New York, 109 - 126.

Sugiura, M. Kawashima, R., Nakamura, K., Okada, K., Kato, T., Nakamura, A., Hatano, K., Itoh, K., Kojima, S., Fukuda, H.: 2000, "Passive and Active Recognition of One's Own Face", Neuroimage 11, 36 - 48.

Takumi, K. and De Boer, R.: 1996, "Self-Assertion Modeled as a Network Repertoire of Multi-Determinant Antibodies", Journal of Theoretical Biology 183, 55 - 66.

Tauber, A.: 1994, The Immune Self: Theory or Metaphor, Cambridge University Press, New York.

Thomas, E. A., Bertrand, P. P., Bornstein, J. C.: 1999, "Genesis and Role of Coordinated Firing in a Feedforward Network: a Model Study of the Enteric Nervous System", Neuroscience 93 (4), 1525 - 1537.

Tomita, M. et al. (1999) "E-CELL: Software Environment for Whole-Cell Simulation", Bioinformatics 15 (1), 72 - 84.

van Inwagen, P.: 1990, Material Beings, Cornell University Press, Ithaca, NY, sec. 15.

Varela, F. and Coutinho, A.: 1991, "Second Generation Immune Networks", Immunology Today 12, 159 - 166.

Varela, F., et al.: 1988, "Cognitive Networks: Immune, Neural, and Otherwise", Theoretical Immunology (Part 2; Vol. 3), 359-375.

Wallace, J. and MacCrimmon, D.: 1990, "Hyperthyroidism: Cognitive and Emotional Factors", in C. Holmes (ed.) Psychoneuroendocrinology: Brain, Behavior, and Hormonal Interactions, Springer-Verlag, New York, 323-343.

Wedekind, C. \& Furi, S.: 1997, "Body Odour Preferences in Men and Women: Do they Aim for Specific MHC Combinations or Simple Heterozygosity?", Proceedings of the Royal Society of London (Series B) 264, 1471 - 1479. 
White, R. J. et al.: 1996, "The Isolation and Transplantation of the Brain", Neurological Research 18 (3), 194 - 203.

White, R. J., Wolin, L. R., Massopust, L., Taslitz, N., Verdura, J.: 1971, "Cephalic Exchange Transplantation in the Monkey", Surgery 70 (1), 135-139.

White, R. J.: 1999, "Head Transplants", Scientific American 10 (3), 24 - 26.

Wilkes, K.: 1988, Real People: Personal Identity without Thought Experiments, Oxford University Press, New York.

Yockey, H.: 1992, Information Theory and Molecular Biology, Cambridge University Press, New York.

Yuh, C.-H. et al.: 1998, "Genomic cis-Regulatory Logic: Experimental and Computational Analysis of a Sea Urchin Gene", Science 279 (20 March), 1896 1902. 